\title{
Redshift measurement of Fermi Blazars for the Cherenkov Telescope Array
}

\author{
P. Goldoni ${ }^{* 1}$, S. Pita ${ }^{1}$, C. Boisson ${ }^{2}$, G. Cotter ${ }^{3}$, D.A. Williams ${ }^{4}$ and E. Lindfors ${ }^{5}$ for \\ the CTA Consortium \\ ${ }^{1}$ APC, Univ Paris Diderot, CNRS/IN2P3, CEA/Irfu, Obs de Paris, Sorbonne Paris Cité, France, \\ 10 rue Alice Domon et Leonie Duquet, 75205, Paris Cedex 13, France \\ 2 Observatoire de Paris, LUTH, CNRS, Université Paris Diderot, PSL, France \\ ${ }^{3}$ Department of Astrophysics, University of Oxford OX1 3RH, UK \\ ${ }^{4}$ University of California, Santa Cruz, CA 95064, USA \\ 5 Tuorla Observatory, University of Turku, Finland \\ E-mail: goldoni@apc.univ-paris7.fr, pita@apc.univ-paris7.fr, \\ catherine.boisson@obspm.fr, Garret. Cotterephysics.ox.ac.uk, \\ daw@ucsc.edu, elilin@utu.fi
}

\begin{abstract}
Blazars are active galactic nuclei, and the most numerous High Energy (HE) and Very High Energy (VHE) $\gamma$-ray emitters. Their optical emission is often dominated by non-thermal, and, in the case of BL Lacs, featureless continuum radiation. This renders the determination of their redshift extremely difficult. Indeed, as of today only about $50 \%$ of $\gamma$-ray blazars have a measured spectroscopic redshift. The knowledge of redshift is fundamental because it allows the precise modeling of the VHE emission and also of its interaction with the extragalactic background light (EBL). The beginning of the Cherenkov Telescope Array (CTA) operations in the near future will allow the detection of several hundreds of new BL Lacs. Using the first Fermi catalogue of sources above $10 \mathrm{GeV}$ (1FHL), we performed simulations which demonstrate that at least half of the 1FHL BL Lacs detectable by CTA will not have a measured redshift. Indeed the organization of observing campaigns to measure the redshift of these blazars has been recognized as a necessary support for the AGN Key Science Project of CTA. Taking advantage of the recent success of an X-shooter GTO observing campaign, we thus devised an observing campaign to measure the redshifts of as many as possible of these candidates. The main characteristic of this campaign with respect to previous ones will be the use of higher resolution spectrographs and of 8 meter class telescopes. We are starting submitting proposals for these observations. In this paper we will briefly describe the selection of the candidates, the characteristics of these observation and the expected results.
\end{abstract}

The 34th International Cosmic Ray Conference,

30 July- 6 August, 2015

The Hague, The Netherlands

\footnotetext{
* Speaker.

${ }^{\dagger}$ Full consortium author list at http://cta-observatory.org
} 


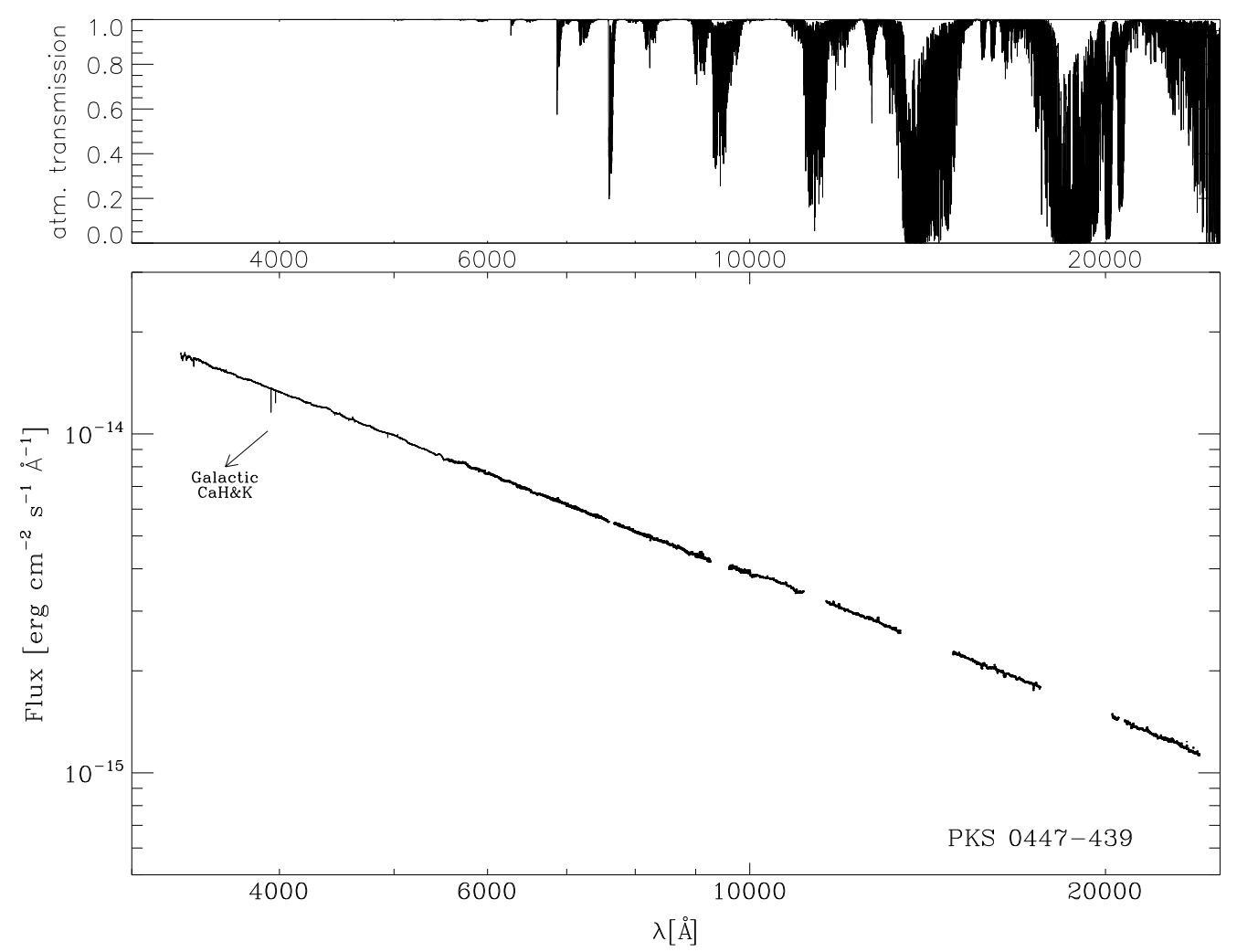

Figure 1: UV to NIR spectrum of PKS 0477-439 (from [13]) obtained with X-shooter, the non thermal continuum radiation produces a power law spectrum. The only spectral feature of astrophysical origin detected in the spectrum is the $\mathrm{Ca} \mathrm{H} \& \mathrm{~K}$ absorption from the interstellar medium of the Milky Way. In the upper panel we show the typical Earth atmospheric transmission from Paranal which imprints telluric absorption features in the spectrum.

\section{Introduction}

Blazars are a type of active galactic nuclei possessing relativistic plasma jets pointing toward the observer (see [15] for a review). They are the most numerous $\gamma$-ray emitters in the $\mathrm{MeV}$ to $\mathrm{TeV}$ range. The majority of the energy emitted comes from the radiation of particles accelerated in these jets, which is further Doppler boosted along the line of sight. This tends to hide the spectral signature of the host galaxy and renders, especially for BL lacs objects, the determination of the redshift difficult. Their spectral energy distribution is characterized by two bumps, which are generally explained in the framework of one-zone leptonic models referred to as Synchrotron selfCompton (SSC) )[5], [6]). Alternatively the second peak may be produced by hadronic processes often associated to pion decay (e.g. [9]). In this case BL Lacs may be a source of high energy cosmic rays and/or neutrinos [2]. It is also possible that the radiation is produced at great distance from the BL Lac, if particles from the jet trigger cascades in the intergalactic space interacting with the CMB or the EBL [3].

At energies greater than $100 \mathrm{GeV}$ or so VHE $\gamma$-rays are heavily affected by the interaction with the Extragalactic Background Light (EBL) [7]. The EBL is the optical to NIR radiation released 
by the stars and galaxies throughout the evolution of the Universe. The $\mathrm{TeV}$ photons emitted by the BL Lacs annihilate with the optical ones ( $\gamma-\gamma$ interaction) producing electron-positron pairs. The resulting $\gamma$-ray opacity is strongly dependent on the distance to the source and dramatically softens the $\mathrm{TeV}$ spectra for energies higher than $1 \mathrm{TeV}$ and redshift $\gtrsim 0.2$ [10]. This effect makes it very difficult to detect BL Lacs at $\mathrm{z} \gtrsim 0.5$. Conversely, if a complete modeling of the BL Lac radiation and of the EBL absorption can be achieved, the EBL properties can be constrained [8].

It is therefore crucial to measure the redshift of a BL Lac. Unfortunately, the continuum optical/NIR emission of the jet is often much stronger than the one of the host galaxy. Indeed, BL Lacs were defined as sources with emission lines with equivalent width (EW) smaller than 5 $\AA$ [15]. An example of the featureless continuum of a bright BL Lac is shown in Figure 1. If the host galaxy is not detected, it is impossible to determine the redshift (and therefore the distance) of the BL Lac from the spectrum. This prevents not only the determination of the amount of line-of-sight absorption but also the measurement of the luminosity of the source.

Several deep observing campaigns have been organized in order to measure BL Lac redshifts, but in each campaign, for a significant fraction of BL Lacs (e.g. $~ 36 \%$ in [12]), no spectral feature could be detected. After the very extensive recent survey by [14], only $\sim 44 \%$ of Fermi BL Lacs have a spectroscopic redshift. The lack of information on redshift (and therefore luminosity) of a great fraction of the sources very strongly biases population studies. This situation is more disturbing in view of the imminent start of the operations of CTA.

As BL Lacs are the dominant source class in the TeV extragalactic sky, it is expected that CTA will discover hundreds of new BL Lacs, several of them without redshift. In order to estimate the number of them, we used the results of the 1FHL Fermi catalog [1] to simulate CTA observations. The results show that about half of the newly discovered VHE BL Lacs will have no measured redshift. A deep search for their redshift is well justified, but from the above considerations, the observation method should be chosen carefully.

Recently [13] have observed a small sample of $\gamma$-ray BL Lacs using the high spectral resolution spectrograph X-shooter at the ESO Very Large Telescope (VLT). They have shown that, under these conditions, a one hour observation is sufficient to find or constrain the redshift for most of these objects. Their sample included five objects observed also by [14], who measured a redshift for only one of them. Conversely [13] measured three redshifts and two strict lower limits from line of sight absorption systems. These encouraging results prompted us to organize a program to measure the redshift of BL Lacs candidates for CTA detection.

In the following we describe the observations performed by [13], the simulations that allowed us to select our sample and the plan of the long term observing campaign.

\section{Previous spectroscopic observations}

In their study of eight HE BL Lacs with unknown or uncertain redshift, [13] determined the redshift of five of them and constrained the redshift of two others through the detection of intervening absorbing systems. The observations were performed with the $\mathrm{X}$-shooter spectrograph [16] at the VLT. X-shooter is a high resolution single object spectrograph sensitive to radiation from $3000 \AA$ to $24000 \AA$. The radiation from the source is split by two dichroics in three arms, UVB 
(sensitive from $3000 \AA$ to $5600 \AA$ ), VIS (sensitive from $5500 \AA$ to $10200 \AA$ ) and NIR (sensitive from $10000 \AA$ to $24000 \AA$ ).

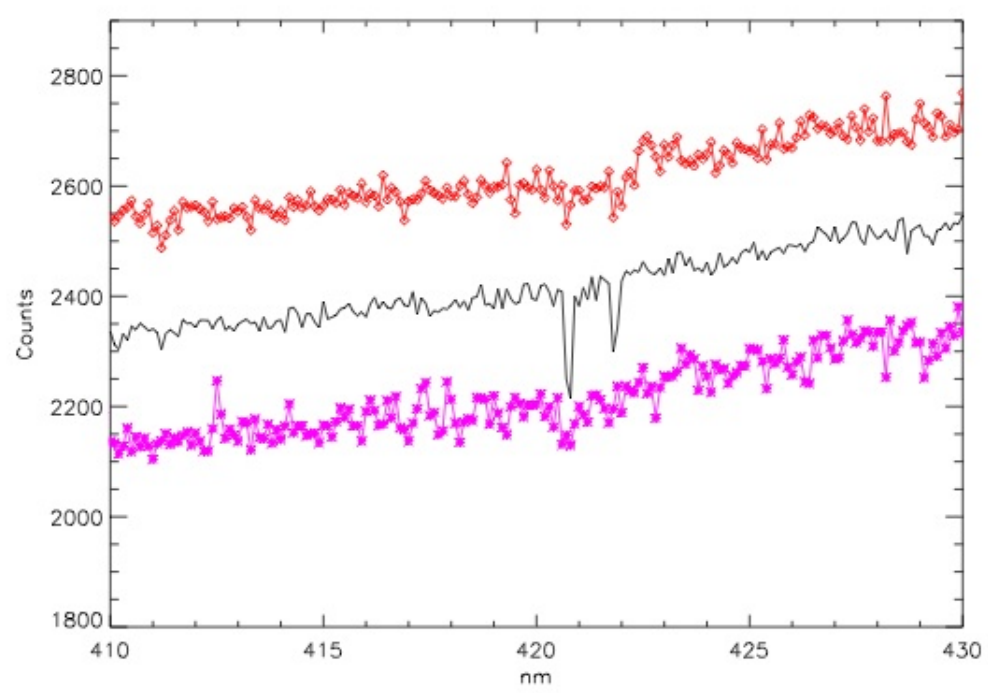

Figure 2: We present here the region of the spectrum KUV 0033-1198 containing the MgII doublet absorption at $\mathrm{z}=0.505$ as seen in the original $(\mathrm{R}=10000) \mathrm{X}$-shooter spectrum [13] (center, black line) and the same spectrum convolved to reduce its spectral resolution to $\sim 600$ (upper, connected red squares) and $\sim$ 1100 (lower, connected magenta asterisks). The red and magenta spectrum are artificially offset for display purposes. It is apparent that identifying the doublet becomes much more difficult at lower resolution even if the signal to noise ratio is the same.

Examination of the results showed that the main factors in this success were: the high spectral resolution ( $\left.\mathrm{R}=\frac{\lambda}{\Delta \lambda} \sim 10000\right)$, the sensitivity and wavelength range of the instrument and the good observing conditions. In particular the spectral resolution is very effective for the detection of narrow spectral features such as the ones due to intervening absorption systems (Figure 2). The resolution can also be very helpful for the correct identification of spectral features produced by the Earth's atmosphere (see upper box in Figure 1).

The average $\mathrm{S} / \mathrm{N}$ per pixel reached in these observations was $\sim 100$ in the UVB arm, $\sim 80$ in the VIS arm and $\sim 40$ in the NIR arm, the trend of lower $\mathrm{S} / \mathrm{N}$ with wavelength being mainly due to the spectral shape (see Figure 1). This high S/N combined with the unprecedented wavelength range permitted enough features to firmly determine the redshift to be found. Depending on the source, they detected between two and six features, always including a doublet, see Table 1 for the details. Moreover, the features that allowed the determination of the redshift were already detected after one hour of exposure. Finally, despite reaching a very high S/N, no feature linked to the host galaxy could be detected in two sources: PKS 0477-439 and KUV 00311-1198. This suggests that it is not worthwhile to observe for very long exposure times as for some sources the spectral features cannot be detected even in these conditions. 
Table 1: List of main features detected for each source in [13]. The columns contain: (1) the name of the source, (2) the redshift, (3) the name of the absorption and emission features detected. Note that Ca II H\&K and Mg II $\lambda 2796 \AA, 2803 \AA$ are doublets, their detection determines the redshift unambiguously. The majority of these features were detected after one hour of exposure.

\begin{tabular}{|c|c|c|}
\hline Source & Redshift & Features \\
\hline BZB J0238-3116 & 0.2329 & Ca II H\&K, H $\delta$, Ca I G, Na I D, [NII] $\lambda 6548 \AA$, [NII] $\lambda 6583 \AA$ \\
\hline BZB J0543-5532 & 0.273 & $\mathrm{Ca}$ II H\&K, Na I D \\
\hline BZB J0505+0415 & 0.424 & Ca II H\&K, Mgb, CaI+FeI, Na I D \\
\hline RBS 334 & 0.411 & Ca II H\&K, Ca I G, CaI+FeI, Na I D, Ca triplet \\
\hline PKS 0301-243 & 0.2657 & $\begin{array}{l}{[\mathrm{OII}] \lambda 3727 \AA,[\mathrm{OIII}] \lambda 4959 \AA,[\mathrm{OIII}] \lambda 5007 \AA} \\
{[\mathrm{NII}] \lambda 6548 \AA, \mathrm{H} \alpha,[\mathrm{NII}] \lambda 6583 \AA}\end{array}$ \\
\hline KUV 00311-1938 & $0.506 \leq \mathrm{z} \leq 1.54$ & $\operatorname{Mg}$ II $\lambda 2796 \AA, 2803 \AA$ \\
\hline BZB J0816-1311 & $0.288 \leq \mathrm{z} \leq 1.56$ & Three Mg II $\lambda 2796 \AA ̊, 2803 \AA$ at different redshifts \\
\hline
\end{tabular}

\section{Sample Selection}

For our observing program, we selected the most promising candidates for an early detection by CTA using the recently published 1FHL catalog [1], which provides a list of blazars detected by the Fermi-LAT space telescope above $10 \mathrm{GeV}$ during the three years period from 2008 to 2011. This selection on the photon energy allows to cull the harder and brighter sources detected by Fermi. Simple extrapolation of their spectra, taking into account the effect of EBL absorption (see below for details), show that these sources tend to be good candidates for detection in the VHE domain.

Out of 514 sources detected at energies higher than $10 \mathrm{GeV}$, the 1FHL catalog contains $259 \mathrm{BL}$ Lacs and 58 unidentified AGNs (AGUs), a large fraction of which could be BL Lacs. The 1FHL catalog also lists a redshift value (or the lack of it) for all its extragalactic entries. We corrected their lists using the more recent results of [13]. After these corrections, 131 BL Lacs and 9 AGUs in the 1FHL catalog have an associated redshift, while 128 BL Lacs and 49 AGUs do not,

For this project 275 AGNs not yet detected at VHE were selected. Of these, 110 are BL Lacs and AGUs with redshift (with $<\mathrm{z}>=0.33$ ), 165 are BL Lacs and AGUs without redshift.

Taking the source spectral parameters from the 1FHL catalog implies that we are using the average spectral parameters of the sources and therefore these simulations do not consider flaring episodes. We also make the hypothesis that the average flux of the selected sources during the 1FHL catalog integration is representative of their flux in the CTA era.

For the sources for which the redshift is unknown, in order to simulate the EBL absorption, a redshift has to be assumed. For simplicity we used the same redshift value for all of them. In the survey of [14], the average redshift is 0.33 , while the average lower limit to the redshift from the detection of absorption systems is 0.7 . Accordingly, we choose three values for our simulations: $0.3,0.6$ and 1 . The first choice can be considered slightly optimistic, the second conservative and the third pessimistic, The spectra were then extrapolated into the CTA range applying the EBL absorption from the models of [4].

For all the sources, with known and unknown redshift, we simulated the CTA detection using 
CTA response functions computed by the consortium. As these response functions have been determined for zenith angle $20^{\circ}$, we put a filter on declination $\left(-55^{\circ},+60^{\circ}\right)$ considering $\pm 25^{\circ}$ around the South (Aar) and the North (Tenerife) sites for which the responses have been computed. This cuts 17 sources with redshift and 27 sources without redshift from the sample, leaving us with 93 and 138 sources respectively.

The exposure time was chosen as to achieve a detection level larger than $5 \sigma$ and a $\gamma$-ray excess of more than 300 source events. The results are summarized in Table 2 in bins of 10 hours.

\begin{tabular}{cccccccc}
\hline$<\mathrm{z}>$ & $<10 \mathrm{~h}$ & $10-20 \mathrm{~h}$ & $20-30 \mathrm{~h}$ & $30-40 \mathrm{~h}$ & $40-50 \mathrm{~h}$ & $>50 \mathrm{~h}$ & Total \\
\hline $\mathbf{0 . 3 3}$ & $\mathbf{2 9}$ & $\mathbf{6}$ & $\mathbf{7}$ & $\mathbf{6}$ & $\mathbf{0}$ & $\mathbf{4 5}$ & $\mathbf{9 3}$ \\
\hline \hline 0.3 & 32 & 23 & 9 & 10 & 2 & 62 & 138 \\
\hline 0.6 & 11 & 11 & 10 & 9 & 8 & 89 & 138 \\
\hline 1 & 1 & 2 & 8 & 1 & 1 & 125 & 138 \\
\hline \hline
\end{tabular}

Table 2: Number of sources detected with $>5 \sigma$ significance and more than 300 counts for different CTA exposure times in 10 hours bins. The first line in boldface lists the results for sources with known redshift. The other lines list the results for sources with unknown redshifts for which a redshift of respectively 0.3 , 0.6 and 1 have been assumed.

From the results in the table it appears that, for $<\mathrm{z}>\leq 0.6$ with rather short observations $(\leq$ 20 hours) at least 22 BL Lacs could be reliably detected. Moreover, it is clear that, under fair assumptions, a considerable number of BL Lacs without redshift will be detected, comparable to the numbers of BL Lacs with known redshift.

Details of the simulations will be refined following new results from Fermi and updated CTA response functions as well as new $\mathrm{TeV}$ blazar detection or publications of new redshift determinations. The list of sources will thus be continuously evolving in the coming years

\section{Observing campaign definition}

We have shown that a program to observe $\gamma$-ray BL Lacs optical counterparts with a high spectral resolution $(\mathrm{R} \sim 10000)$ spectrograph at 8 meter class telescope can consideraby expand the number of $\gamma$-ray BL Lacs with known redshift. The selected sources are evenly distributed in the Northern and Southern Hemisperes. However, X-shooter, being installed at VLT, cannot observe them all. Nonetheless, while X-shooter is a unique instrument due to its NIR arm, similar instruments in the UVB to VIS range are installed in some of the World's major telescopes (e.g. ESI at Keck). This survey can therefore be pursued in the Northern hemisphere by using those instruments.

Within the CTA consortium, which is a large international collaboration, access to other facilities is possible and a sustainable observing campaign is being built. Our redshift determination collaboration is open to further members who are experienced in this kind of research and have access to other world class spectrographs similar to the ones previously mentioned.

As a start, a subsample of the most promising 80 sources, equally distributed between Northern and Southern hemispheres was extracted from our simulations. We are submitting proposals to ESO to observe with X-shooter at VLT (Southern Hemisphere) and to the W.M. Keck observatory 
to observe with ESI at Keck (Northern Hemisphere). The goal is to observe about 20 sources per year (10 from the Southern hemisphere and 10 from the Northern Hemisphere). However a key objective is to have a sensitivity as uniform as possible for the whole sample. Therefore, in case of loss of observing time due to technical problems or bad weather, some sources may be reobserved at a later time.

\section{Acknowledgments}

We gratefully acknowledge support from the agencies and organizations listed under Funding Agencies at this website: http://www.cta-observatory.org/.

\section{References}

[1] Ackermann, M., Ajello, M., Allafort, A. et al., The First Fermi-LAT Catalog of Sources above 10 GeV, ApJS 20934 [astro-ph/1306.6772]

[2] Dermer, C. D. \& Atoyan, A., Prospects for detecting high-energy neutrinos from Blazars, in proceedings of 27th International Cosmic Ray Conference, 07-15 August, 2001, Hamburg, Germany, 31149 [astro-ph/0107200]

[3] Essey, W. \& Kusenko, A., A new interpretation of the gamma-ray observations of distant active galactic nuclei, Astroparticle Physics 3381 [astro-ph/0905.1162]

[4] Franceschini, A., Rodighiero, G., Vaccari, M., Extragalactic optical-infrared background radiation, its time evolution and the cosmic photon-photon opacity, A\&A 487837 [astro-ph/0805.1841]

[5] Ginzburg, V.L. \& Syrovatskii, S.I., Cosmic Magnetobremsstrahlung (synchrotron Radiation), ARA\&A 3297

[6] Ghisellini, G. \& Maraschi, L., Bulk acceleration in relativistic jets and the spectral properties of blazars, ApJ 340181

[7] Hauser, M.G. \& Dwek, E., The Cosmic Infrared Background: Measurements and Implications, ARA\&A 39249 [astro-ph/0105539]

[8] HESS collaboration, Measurement of the extragalactic background light imprint on the spectra of the brightest blazars observed with H.E.S.S., A\&A 550 A4 [astro-ph/1212.3409]

[9] Mannheim, K., The proton blazar, A\&A 26967

[10] Mazin, D., Raue, M., Behera, B. et al., Potential of EBL and cosmology studies with the Cherenkov Telescope Array, J. Astroph. Phys 43241 [astro-ph/1303. 7124]

[11] Nolan, P.L., Abdo, A.A., Ackermann, M. et al., Fermi Large Area Telescope Second Source Catalog, ApJS 19931 [astro-ph/1108.1435]

[12] Piranomonte, S., Perri, M., Giommi, P. et al., The sedentary survey of extreme high-energy peaked BL Lacs. III. Results from optical spectroscopy, A\&A 470787 [astro-ph/0704.1729]

[13] Pita, S., Goldoni, P., Boisson, C. et al., Spectroscopy of high-energy BL Lacertae objects with $X$-shooter on the VLT, A\&A 565 A12 [astro-ph/1311.3809]

[14] Shaw, M.S., Romani, R.W., Cotter, G. et al., Spectroscopy of the largest ever $\gamma$-ray selected BL Lac sample, ApJ 764135 [astro-ph/1301.0323] 
[15] Urry, C.M. \& Padovani, P., Unified Schemes for Radio Loud Active Galactic Nuclei, PASP 107803 [astro-ph/9506063]

[16] Vernet, J., Dekker, H., D’Odorico, S. et al., X-shooter, the new wide band intermediate resolution spectrograph at the ESO Very Large Telescope, A\&A 536, A105 [astro-ph/1110.1944] 